\title{
The Association between Aortic Valve Weight, Echocardiographic Indices, and All-Cause Death in 1,046 Patients Undergoing Surgical Aortic Valve Replacement for Aortic Stenosis
}

\author{
Jonathan Weber ${ }^{a} \quad$ Simcha Pollack ${ }^{a}$ Florentina Petillo ${ }^{a}$ Ana Anagnostopoulos ${ }^{a}$ \\ J. Jane Cao ${ }^{a, b}$ Eddy Barasch ${ }^{a, b}$

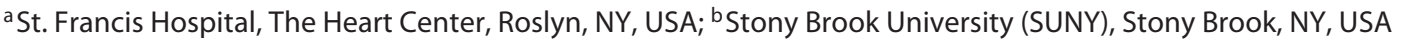

\section{Keywords}

Aortic stenosis - Aortic valve replacement - Aortic valve weight · Survival

\begin{abstract}
Background: Aortic valve weight (AVW), a flow independent measure of aortic stenosis (AS) severity, is reported to have heterogeneous associations with the echocardiographic variables used for AS evaluation. Controversy exists regarding its impact on survival after aortic valve replacement (AVR). Objective: We sought to determine the association between AVW with echocardiographic measures of AS severity and all-cause mortality after surgical AVR. Methods: One thousand and forty-six consecutive patients underwent surgical AVR for AS, the excised valves were weighed, and an echocardiogram was done before surgery. Results: Males had heavier valves than females, for both absolute and body surface are (BSA)-indexed values ( $2.78 \pm 1.23$ vs. $2.08 \pm 0.68$ $\mathrm{g}, p<0.001$; and $1.38 \pm 0.61$ vs. $1.19 \pm 0.41 \mathrm{~g} / \mathrm{m}^{2}, p<0.001$, respectively). In a restricted cohort of 634 patients with isolated severe AS and normal ejection fraction, the correlations of AVW with echocardiographic variables of AS were modest, the strongest being with the dimensionless index $(r=-0.27$ and -0.26 for male and female, both $p<0.01)$. Strat-
\end{abstract}

ified by stroke volume index and mean gradient (MG), no associations were found in the low-gradient groups (i.e., MG $<40 \mathrm{~mm} \mathrm{Hg}$ ). At a median follow-up of 3.5 years, there were only 244 deaths in the entire cohort. Mortality was not related to AVW, except in females who displayed an inverse relationship ( $\mathrm{HR}=0.67 ; 95 \% \mathrm{Cl} 0.47-0.95)$ only when it was analyzed as a continuous variable. Conclusions: The weak correlation between AVW with the echocardiographic indices of AS may reflect its complex pathophysiology, heterogeneous hemodynamics, and possible pitfalls in the current echocardiographic methods used in clinical practice. The prognostic value of AVW after AVR warrants further evaluation.

(c) 2020 S. Karger AG, Basel

\section{Introduction}

Aortic valve calcification (AVC) is the end result of a lengthy process involving genetic [1-3], epigenetic [4], inflammatory [5], humoral [6,7], and hemodynamic factors [5], culminating with the ectopic deposition of calcium and phosphate on the aortic cusps, which in turn leads to increased valve stiffness, reduced cusp excursion, and progressive valve orifice-narrowing [8]. Gender differenc-

karger@karger.com
www.karger.com/crd
Karger ${ }^{\prime /}$

Dr. Eddy Barasch, MD

St. Francis Hospital, The Heart Center/SUNY at Stony Brook 100 Port Washington Blvd

Roslyn, NY 11576 (USA)

Eddy.Barasch@chsli.org 
es in the pathogenesis of aortic stenosis (AS) have been identified such that, compared with males, females (with the same hemodynamic severity), have a lower AVC load and more valvular fibrotic changes $[9,10]$. A good correlation between AVC evaluated by multidetector computerized tomography (MDCT) with echocardiographic hemodynamic indices of AS severity has been reported [1113], and a modest correlation when AVC load was determined by the aortic valve weight (AVW) [14-17]. In contrast, the association with aortic valve area (AVA) was much weaker when AVC was determined by both MDVT and AVW $[14,18]$. The strongest correlates among the echocardiographic indices of AS with AVW, a hemodynamic independent measure of AS severity, their level in the 4 hemodynamic groups of patients with severe AS and preserved left ventricular ejection fraction (LVEF), and whether or not valve weight at the time of aortic valve replacement (AVR) has prognostic value, have been less evaluated. The aims of this study were to: (1) determine the relationship between the echocardiographic measures of AS severity and AVW in a selected group of patients with severe isolated AS and normal LVEF, analyzed as a single cohort as well as categorized into 4 hemodynamic groups based on LV stroke volume index (SVi) and mean (transvalvular) gradient (MG), and (2) evaluate the prognostic value of AVW in a large cohort of consecutive patients with severe AS who underwent surgical AVR for AS.

\section{Material and Methods}

\section{Study Population}

Our study involved patients who underwent AVR at our institution from 2011 to 2015 , had their excised valves weighed, and had comprehensive echocardiographic data available. Our primary outcome of interest was all-cause mortality. All patients were included in the survival analysis that examined the relationship between AVW and all-cause mortality. A subgroup analysis to evaluate associations between echocardiographic measures of AS with AVW consisted only of patients with LVEF $>50 \%$, severe or critical AS, and no more than $2+$ aortic and/or mitral regurgitation, mitral stenosis, significant tricuspid regurgitation, or prior prosthetic valve implantation were included. Patients with at least 1 of the exclusion criteria were removed from the subgroup analysis. Demographics and clinical and laboratory data were retrieved from the medical records and are presented in the online supplementary Files (for all online suppl. material, see www.karger.com/doi/10.1159/000505870). Mortality data were extracted from medical records and the National Death Index.

\section{Echocardiography}

Transthoracic echocardiography (TTE) and intraoperative transesophageal echocardiography (TEE) were performed using
Phillips IE 33 and Phillips Sonos 7500 scanners (Amsterdam, The Netherlands). The echocardiographic data were taken in $59 \%$ of patients from the TEE performed before AVR, and from the TTE performed within 6 months of surgery in the rest of the patients. All the variables were remeasured off-line by a single analyst (F.P.), using a dedicated echocardiographic reading station (Agfa/Heart Lab). For each Doppler measurement, for patients in sinus rhythm, 3 cardiac cycles were averaged, and for those in atrial fibrillation, 5 cycles. Severe AS was diagnosed according to the contemporary guidelines [19]. The highest transvalvular velocity was chosen from the standard 4 windows as recommended. The equations used for the determination of AS severity and vascular load are presented in the online supplementary Files.

\section{Pathology}

The excised aortic valves were immersed in a container filled with formaldehyde and transported to the pathology lab. The valve was removed from the container, placed on absorbing paper to absorb excess formaldehyde, and then weighed by the pathologist (A.A.) on an electronic scale (Sartorius Type 1404) with an accuracy of $0.01 \mathrm{~g}$.

\section{Statistical Analysis}

Univariate measures were reported as mean (standard deviation) or $n(\%)$. The primary correlates of AVW were echocardiographic measures of AS severity. Other covariates utilized in the analysis included past medical history, medications at the time of TEE, concurrent coronary artery bypass graft surgery (CABG), demographic information, and other echocardiographic measures of heart function. Bivariate analyses were performed using Student's $t$ test or $\chi^{2}$ test as appropriate. Pearson's correlations (with Fisher's $z$ transformation) of AVW and the primary echocardiographic measures of AS severity were calculated in a restricted subsample of participants with severe isolated AS and LVEF $\geq 50 \%$, all adjusted for age, gender, and stroke volume. Logistic regression (with Youden's index) was used to create gender-specific cutoffs for AVW by predicting severe AS (as defined by $\mathrm{MG} \pm 40 \mathrm{~mm} \mathrm{Hg}$ ) [19] which were then applied to survival models. All-cause mortality was our primary outcome of interest. Unadjusted survival analyses were performed using Kaplan-Meier curves and the log-rank test. Survival hazards were calculated using the Cox proportional hazards models. Three types of models were created: (1) unadjusted models looking at the association between AVW and all-cause mortality; (2) model $1+$ age, gender, and body surface area (BSA); and (3) model $2+$ a history of CABG, congestive heart failure (CHF), LV stroke volume, chronic kidney disease, hypertension, diabetes, and LVEF. Variables were chosen using the disjunctive cause criterion outlined previously [20]. Since gender differences have been found with the effects of AVW on mortality, we calculated survival models stratified by gender. All analyses were performed using SAS v9.4 (Cary, NC, USA).

\section{Results}

Of the 1,616 patients who underwent AVR at our institution from 2011 to $2015,1,046$ had their excised valve weighed and available comprehensive echocardiographic 
Fig. 1. Patient selection. The survival analysis cohort consisted of 1,046 patients who underwent AVR and had their valves weighed. After restriction, 634 patients with severe isolated AS were included in the subgroup analysis.

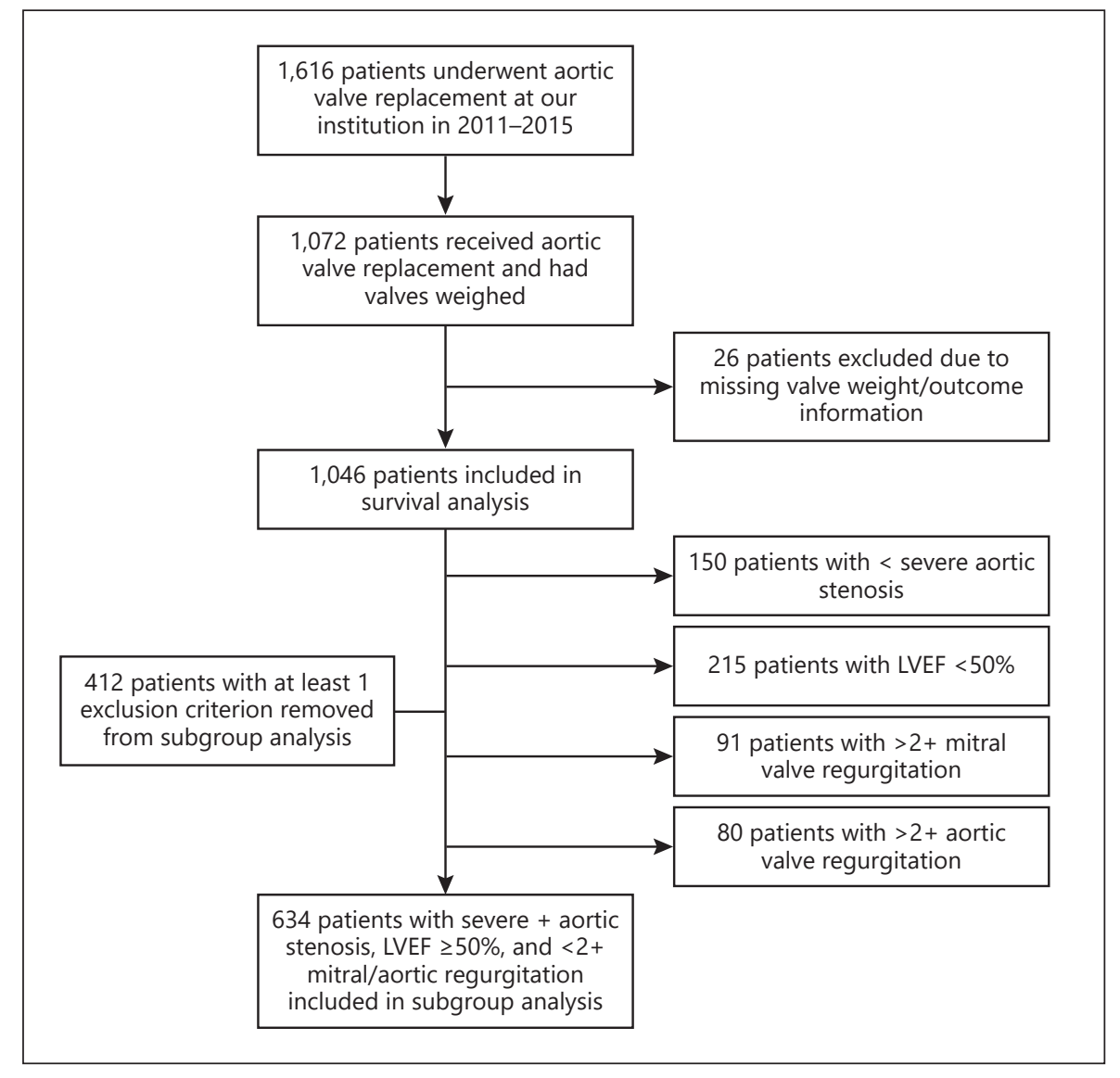

Table 1. Patient characteristics

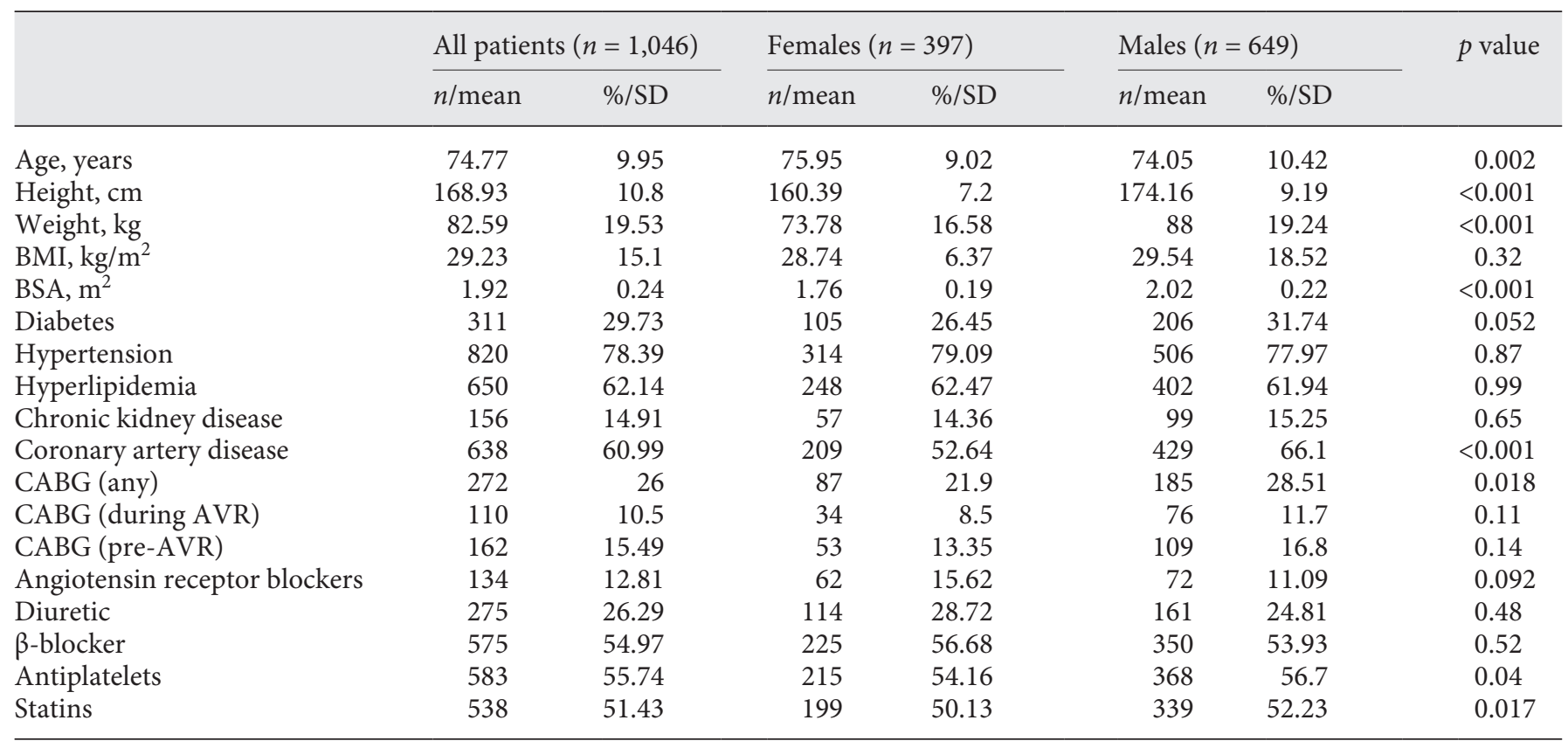

BMI, body mass index; BSA, body surface area; CABG, coronary artery bypass surgery; AVR, aortic valve replacement. 
Table 2. Echocardiographic data

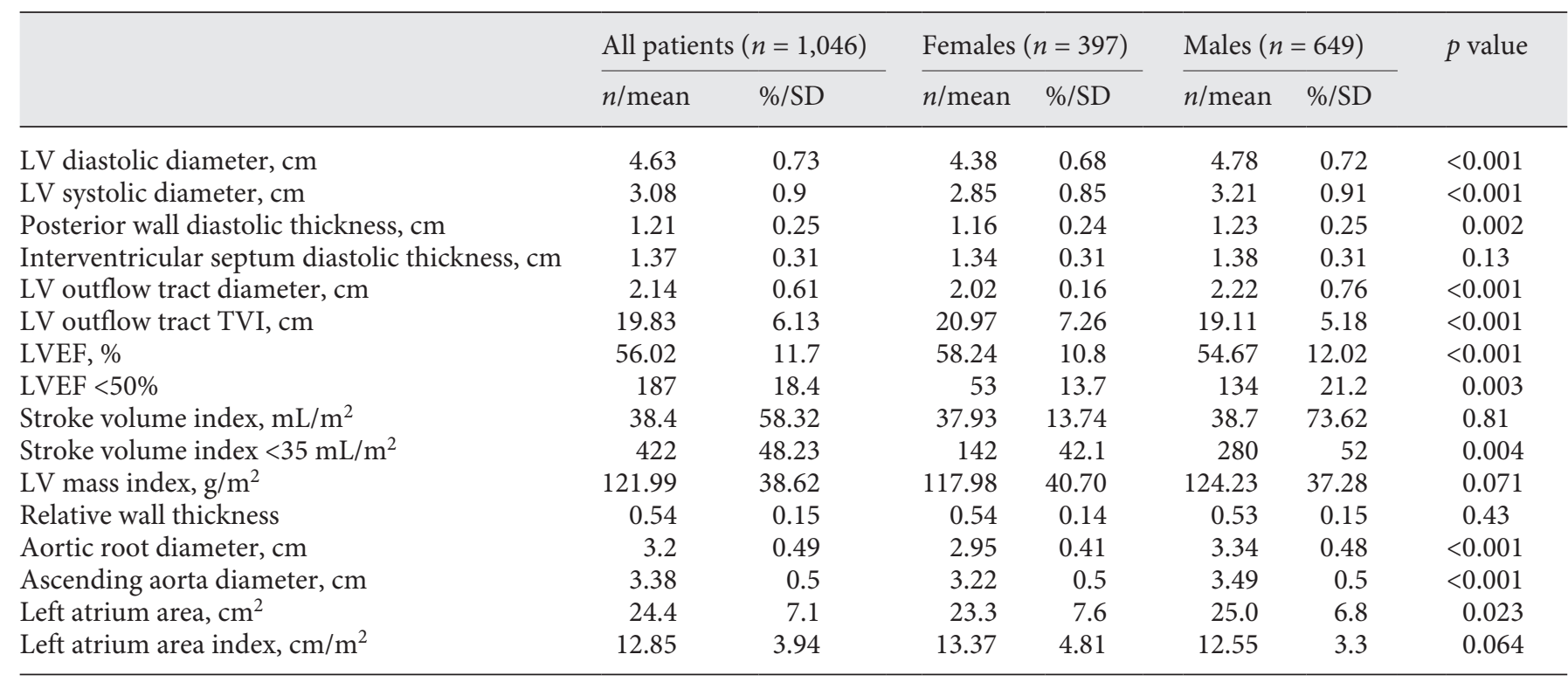

LV, left ventricular; TVI, time velocity integral; LVEF, LV ejection fraction.

data. This sample was thus used for the survival analyses. In order to evaluate associations between echocardiographic measures of AS with AVW, only 634 patients were included who had LVEF $>50 \%$, severe or critical AS, and no more than $2+$ aortic and/or mitral regurgitation, mitral stenosis, significant tricuspid regurgitation, or prior prosthetic valve implantation. All 1,046 patients were included in the survival analyses. Our criteria for patient selection are outlined in Figure 1. The 1,046 patients who underwent AVR for AS had a mean age of $74.8 \pm 10$ years and $397(40 \%)$ were female (Table 1). Of the entire cohort, 877 (84\%) had severe AS, 139 (14\%) had moderate AS, and 30 (4\%) patients had less than moderate AS. All patients with less than moderateto-severe AS had either concurrent CABG, mitral valve replacement, or ascending aortic aneurysm repair. Of the patients with severe AS, 47 also had aortic regurgitation $>2+$, and 67 had $>2+$ mitral regurgitation. At the time of AVR, 78 patients underwent mitral valve repair or replacement and 110 underwent concurrent CABG; 99 of these had severe AS and 11 had moderate AS. The vast majority (86\%) were in sinus rhythm and $11 \%$ were in atrial fibrillation.

Compared with males, the females were older, had a lower BSA, a lower prevalence of diabetes, coronary artery disease, and CABG, and a lower utilization of antiplatelet agents and statins (Table 1). They also had smaller left ventricles and LV outflow tract (LVOT) diameters; a trend of a smaller LV mass index, a higher LVEF, and a lower prev- alence of SVi $<35 \%$; and a higher dimensionless index (DOI), mean left atrial pressure, and systemic vascular resistance (Tables 2, 3). At pathology, mean AVW was $2.5 \pm$ $1.1 \mathrm{~g}$, (range $0.2-12.1 \mathrm{~g}$, median $2.0 \mathrm{~g}$, interquartile range [IQR] 1-3 g). The AVW in the 649 males ranged from 1.00 to $12.1 \mathrm{~g}$, and the valves in the 397 females ranged from 0.20 to $5.5 \mathrm{~g}$. The males had heavier valves than females in both absolute and BSA-adjusted values $(2.78 \pm 1.23$ vs. 2.08 $\pm 0.68 \mathrm{~g}, p<0.001 ; 1.38 \pm 0.61$ vs. $1.19 \pm 0.41 \mathrm{~g} / \mathrm{m}^{2}, p<$ 0.001 ) but similar values of AVW/aortic annular area (Table 3). Based on the findings of a logistic regression model predicting severe AS ( $M G \geq 40 \mathrm{~mm} \mathrm{Hg}$ ), AVW cutoff points were created at $2.64 \mathrm{~g}$ for males and $2.13 \mathrm{~g}$ for females. For the entire cohort, compared with the lighter AVW group, those with heavier valves were younger, had a higher BSA, a higher prevalence of bicuspid valves (BAV), and a lower prevalence of mitral valve surgery, $\mathrm{CABG}$, $\mathrm{CHF}$, and angiotensin receptor blocker utilization (online suppl. Table 1). On echocardiography, 102 (10\%) patients had BAV with a similar prevalence in both genders. Compared with tricuspid aortic valves, the BAV were heavier in absolute and relative values (online suppl. Table 2).

\section{The Association of AVW with Echocardiographic Measures of AS Severity}

A restricted subset of 634 patients with severe isolated AS, an LVEF $>50 \%$, no more than $2+$ aortic and/ 
Table 3. LV diastolic function, aortic valve anatomy, AS severity, vascular load, and outcome distribution by gender

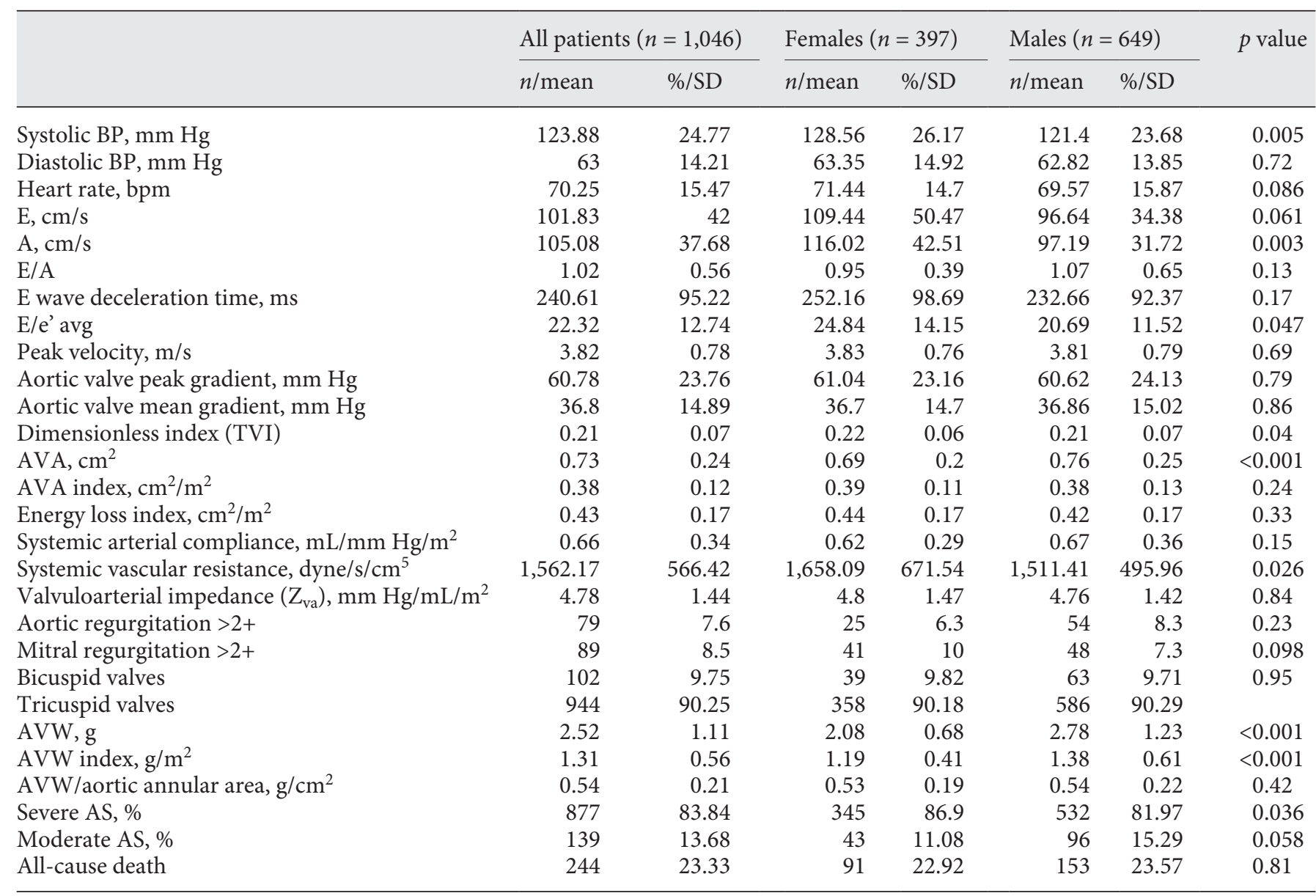

BP, blood pressure; LV, left ventricular; TVI, time velocity integral; AS, aortic stenosis; AVA, aortic valve area; AVW, aortic valve weight.

or mitral regurgitation, mitral stenosis, significant tricuspid regurgitation, or prosthetic valves were used to examine correlations adjusted for age, BSA, and stroke volume between unindexed and indexed AVW with echocardiographic measures of AS severity. To correct for the lack of normal AVW distribution, different transformations of the AVW variable using square root, cube root, and logarithmic transformations were done, with no change in the results. For both genders, the correlation coefficients were modest, and the strongest was between AVW and AVW/BSA with DOI: $r=-0.28$ for both $(95 \%$ CI -0.35 to -0.20 and -0.36 to -0.20 , respectively). Upon stratification by gender (online suppl. Table $3 \mathrm{~A}, \mathrm{~B}$ ), correlations with AVW and AVW/BSA in both genders were very similar whereas those with AVW/aortic annulus area differed, i.e., correlations in males increased modestly while those in females be- came weaker with peak velocity, gradients, DOI, and energy loss index. Although AVW/aortic annulus area did not significantly differ by gender, the association between AS variables and AVW/aortic annulus area appeared to be stronger in males than in females (online suppl. Table 3A, B). Figure 2 illustrates the relationship between AVW (indexed by aortic annular area) and peak gradient.

Finally, we examined AVW in the 4 hemodynamic groups of severe AS with preserved LVEF, based on SVi $\leq />35 \mathrm{~mL} / \mathrm{m}^{2}$; $\mathrm{MG} \leq />40 \mathrm{~mm} \mathrm{Hg}$, i.e., normal flow/high gradient $(\mathrm{NF} / \mathrm{HG})$, normal flow/low gradient $(\mathrm{NF} / \mathrm{LG})$, low flow/high gradient (LF/HG), and low flow/low gradient (LF/LG) (Table 4); and their correlations (Table 5). The patients with NF/HG had the heaviest valves and those with low gradients had the lightest valves. Overall, the correlations remained modest. In the high-gradient 
Fig. 2. The relationship between AVW, indexed by aortic annular area, and aortic peak gradient. Of 634 patients with severe AS, modest correlations were found between echocardiographic indices of AS and $\operatorname{AVW}(r=0.20, p<0.001)$.

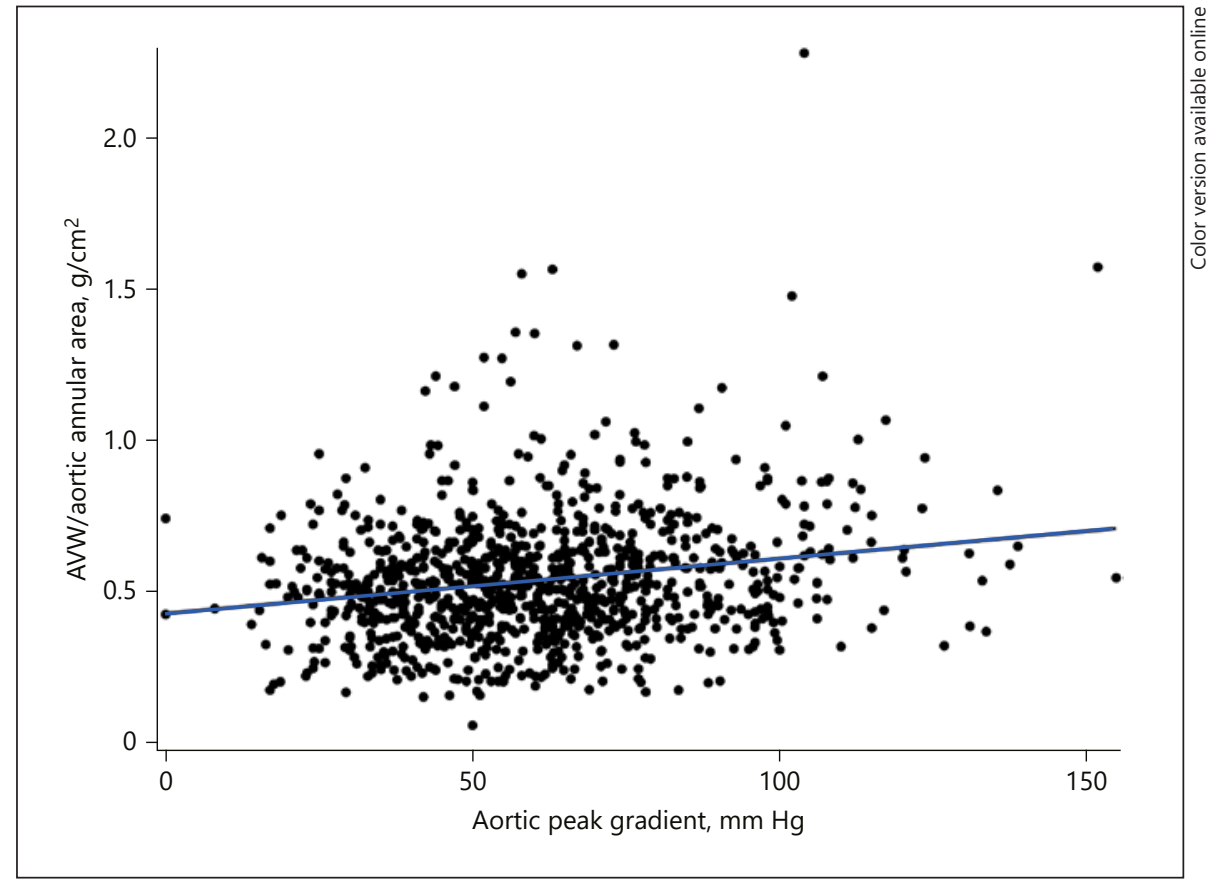

Table 4. Aortic valve weight (AVW) by hemodynamic group

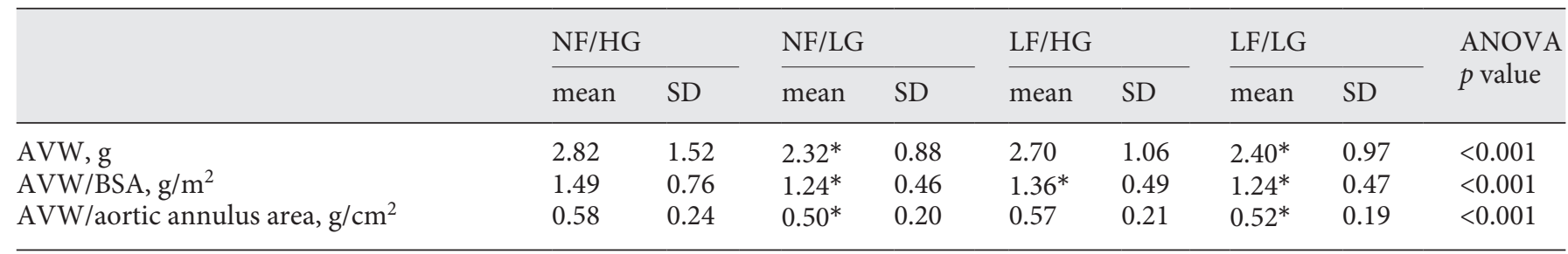

* Different from reference NF/HG group ( $p=0.05$ using Dunnett's test). NF/HG, normal flow/high gradient; NF/LG, normal flow/ low gradient; LF/HG, low flow; high gradient; LF/LG, low flow/low gradient.

groups, values were significant for most indices with AVW/aortic annulus area; with AVW and AVW/BSA, only DOI, AVA, and energy loss index values were significant. In the 2 low-gradient groups, no correlations were found with AVW/aortic annulus area.

\section{The Association of AVW with All-Cause Mortality}

At a median follow-up period of 3.5 years (IQR 2.6-4.7 years), 244 patients had died. Unadjusted Kaplan Meier analysis found similar mortality for both high and low valve weights (Fig. 3, dichotomized valve weight defined as $\pm 2.64 \mathrm{~g}$ for males and $\pm 2.13 \mathrm{~g}$ for females) and gender (online suppl. Fig. 1). Adjusted Cox proportional hazards were done using the entire sample $(n=1,046)$. Three models were created, to estimate survival hazards as a function of AVW taken as a continuous variable and a dichotomized variable stratified by gender at $\pm 2.64 \mathrm{~g}$ for males and $\pm 2.13 \mathrm{~g}$ for females (Table 6). For both genders in all models, when AVW was treated as a categorical variable, and for males only when analyzed as a continuous one, no association with death was found. For females, when AVW was taken as a continuous measure, an inverse association with death was observed (HR 0.67; 95\% CI 0.47-0.95). Sensitivity analyses were performed to establish if the echocardiographic exam method (i.e., TTE or TEE) influenced our conclusions regarding the impact of AVW on survival; no meaningful differences were found. 
Fig. 3. Kaplan-Meier survival estimates of all-cause mortality stratified by valve weight group. A high valve weight was defined as $\geq 2.64 \mathrm{~g}$ for men and $\geq 2.13$ for women. No association was found between valve weight groups and mortality (logrank $p=0.31)$. ${ }^{*}$ Dichotomized at $\pm 2.64 \mathrm{~g}$ for males and $\pm 2.13 \mathrm{~g}$ for females.

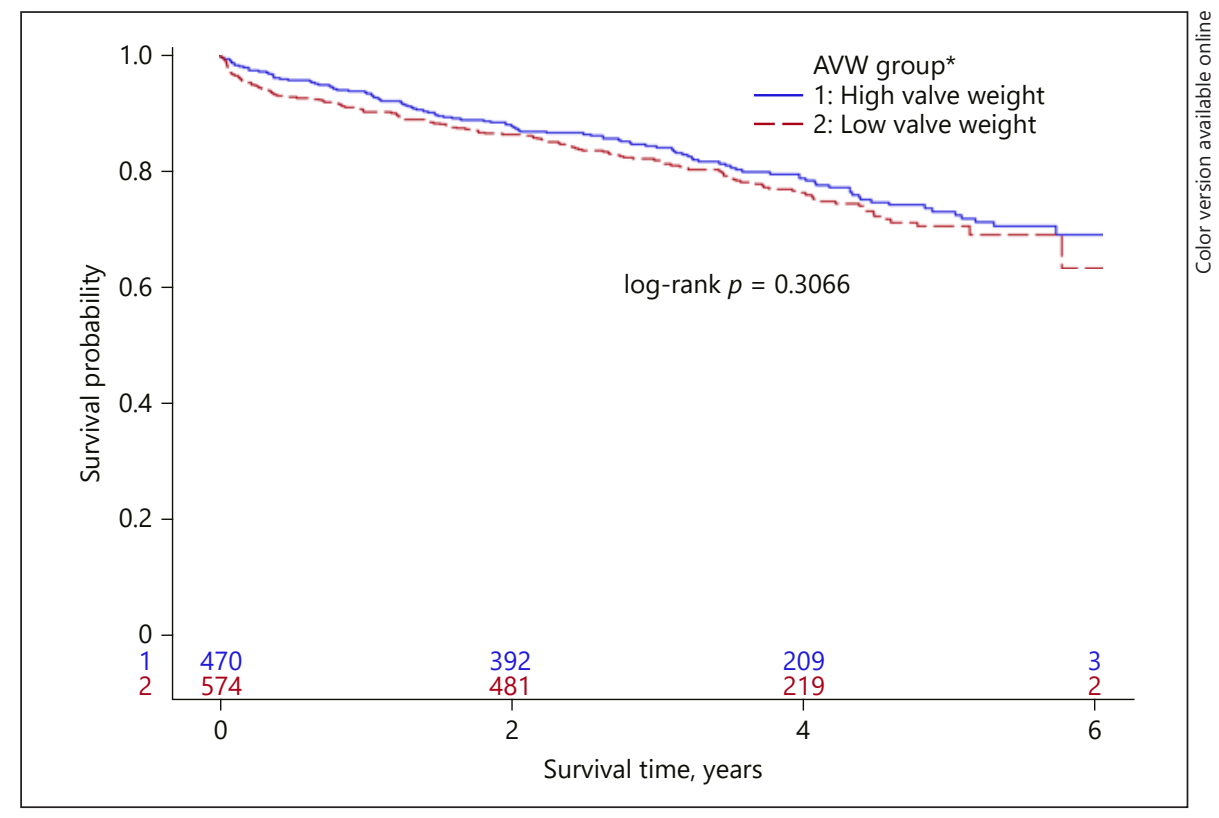

Table 5. Correlations of aortic valve weight with echocardiographic measures of aortic stenosis by hemodynamic group

\begin{tabular}{|c|c|c|c|c|c|c|c|c|c|c|c|c|}
\hline Echo variable & \multicolumn{3}{|c|}{$\mathrm{NF} / \mathrm{HG}$} & \multicolumn{3}{|c|}{ NF/LG } & \multicolumn{3}{|c|}{$\mathrm{LF} / \mathrm{HG}$} & \multicolumn{3}{|l|}{$\mathrm{LF} / \mathrm{LG}$} \\
\hline \multicolumn{13}{|l|}{$A V W$} \\
\hline AV peak gradient & 0.13 & -0.02 & 0.28 & 0.07 & -0.10 & 0.24 & 0.07 & -0.14 & 0.26 & 0.03 & -0.14 & 0.19 \\
\hline AV mean gradient & 0.12 & -0.03 & 0.27 & 0.11 & -0.06 & 0.28 & 0.12 & -0.08 & 0.31 & 0.12 & -0.05 & 0.28 \\
\hline Dimensionless index (TVI) & -0.32 & -0.45 & -0.18 & -0.22 & -0.38 & -0.05 & -0.25 & -0.43 & -0.05 & -0.21 & -0.36 & -0.04 \\
\hline EL index & -0.17 & -0.33 & -0.01 & -0.01 & -0.21 & 0.18 & -0.28 & -0.49 & -0.04 & -0.13 & -0.31 & 0.06 \\
\hline \multicolumn{13}{|l|}{$A V W / B S A$} \\
\hline Peak velocity & 0.12 & -0.04 & 0.26 & 0.08 & -0.10 & 0.25 & 0.07 & -0.13 & 0.27 & -0.01 & -0.18 & 0.15 \\
\hline AV peak gradient & 0.13 & -0.02 & 0.28 & 0.07 & -0.10 & 0.24 & 0.08 & -0.12 & 0.28 & 0.01 & -0.15 & 0.18 \\
\hline AV mean gradient & 0.13 & -0.03 & 0.27 & 0.11 & -0.07 & 0.27 & 0.14 & -0.06 & 0.33 & 0.11 & -0.06 & 0.27 \\
\hline Dimensionless index (TVI) & -0.33 & -0.46 & -0.19 & -0.22 & -0.38 & -0.05 & -0.24 & -0.42 & -0.04 & -0.20 & -0.36 & -0.04 \\
\hline Peak velocity & 0.21 & 0.06 & 0.35 & 0.15 & -0.02 & 0.32 & 0.20 & 0.00 & 0.39 & 0.04 & -0.13 & 0.20 \\
\hline AV peak gradient & 0.22 & 0.07 & 0.36 & 0.14 & -0.03 & 0.31 & 0.23 & 0.03 & 0.41 & 0.07 & -0.10 & 0.23 \\
\hline AV mean gradient & 0.19 & 0.04 & 0.34 & 0.15 & -0.02 & 0.32 & 0.26 & 0.06 & 0.44 & 0.10 & -0.07 & 0.26 \\
\hline Dimensionless index (TVI) & -0.14 & -0.29 & 0.01 & -0.01 & -0.19 & 0.16 & -0.13 & -0.32 & 0.08 & -0.02 & -0.19 & 0.15 \\
\hline AVA & -0.29 & -0.42 & -0.14 & -0.05 & -0.22 & 0.12 & -0.24 & -0.42 & -0.04 & -0.11 & -0.27 & 0.06 \\
\hline AVA index & -0.29 & -0.42 & -0.14 & -0.05 & -0.22 & 0.13 & -0.27 & -0.44 & -0.07 & -0.10 & -0.26 & 0.07 \\
\hline EL index & -0.28 & -0.43 & -0.12 & -0.02 & -0.21 & 0.17 & -0.27 & -0.48 & -0.03 & -0.13 & -0.31 & 0.06 \\
\hline
\end{tabular}

Corr, correlation; LL, lower limit; UL, upper limit; AVW, aortic valve weight; AV, aortic valve; TVI, time velocity integral; AVA, aortic valve area; EL, energy loss; BSA, body surface area. 
Table 6. Cox proportional hazards models estimating hazards of all-cause mortality according to aortic valve weight

\begin{tabular}{|c|c|c|c|c|c|c|c|c|c|c|c|c|}
\hline & \multicolumn{4}{|c|}{ Model $1^{\mathrm{a}}$} & \multicolumn{4}{|c|}{ Model $2^{\mathrm{b}}$} & \multicolumn{4}{|c|}{ Model $3^{c}$} \\
\hline & $H R$ & $L L$ & $U L$ & $p$ value & $H R$ & $L L$ & $U L$ & $p$ value & $H R$ & $L L$ & $U L$ & $p$ value \\
\hline All patients & 0.89 & 0.77 & 1.03 & 0.11 & 0.91 & 0.76 & 1.08 & 0.27 & 0.90 & 0.71 & 1.13 & 0.35 \\
\hline Males & 0.93 & 0.79 & 1.08 & 0.33 & 0.98 & 0.82 & 1.17 & 0.82 & 1.00 & 0.80 & 1.23 & 0.97 \\
\hline Females & 0.73 & 0.54 & 0.99 & 0.046 & 0.72 & 0.52 & 1.00 & 0.049 & 0.67 & 0.47 & 0.95 & 0.023 \\
\hline Males & 0.97 & 0.70 & 1.34 & 0.85 & 1.01 & 0.73 & 1.41 & 0.93 & 0.99 & 0.68 & 1.45 & 0.97 \\
\hline Females & 0.67 & 0.40 & 1.11 & 0.12 & 0.68 & 0.41 & 1.13 & 0.14 & 0.62 & 0.36 & 1.09 & 0.098 \\
\hline
\end{tabular}

HR, hazard ratio; LL, lower limit; UL, upper limit. * Dichotomized at $\pm 2.64 \mathrm{~g}$ for males and $\pm 2.13 \mathrm{~g}$ for females.

${ }^{a}$ Unadjusted model including valve weight alone.

${ }^{\mathrm{b}}$ Includes aortic valve weight in addition to age, gender, and BSA.

${ }^{c}$ Includes all variables in Model $2+$ a history of CABG, heart failure, LV stroke volume, chronic kidney disease, hypertension, diabetes, and LVEF.

\section{Discussion}

The main findings in this large contemporary cohort of patients with AS who underwent surgical AVR are that, in the entire cohort, for a similar degree of AS severity, the AVW values are scattered over a wide range, with females having lighter valves than males, bicuspid valves heavier than tricuspid valves, and significant, albeit modest correlations between AVW and the echocardiographic indices of AS, lacking in the 2 low-gradient groups (NF/ LG and LF/LG). For both genders, when AVW was analyzed as a categorical and for males as a continuous variable, no association with mortality was found, whereas when considered as a continuous measure, an inverse relationship with death was observed in females only.

The large range of AVW in severe AS was previously reported in a study on 499 patients with isolated or mixed aortic valve disease [21]. In contrast to our study, where a large gender difference in the AVW range was observed, in the above-cited study, no gender difference was noted. This discrepancy could be related to differences in patients' characteristics, aortic valve anatomy, and AS type (i.e., isolated or mixed).

The large distribution of AVW in patients with similar AS severity can, in part, be explained by possible measuring errors of the valve hemodynamics and/or area, different fibrotic/calcified valve tissue ratios between males and females, errors in weighing the valve specimens, or other causes. Using the cutoff values of AVW for severe AS in males and females ( 2 and $1.2 \mathrm{~g}$, respectively), a re- cent report indicated 14\% disagreement between the hemodynamic indices of severe AS and AVW in the NF/ $\mathrm{HG}$, and $30 \%$ in the LF/LG group; these results demonstrate the underlying complexity of this relationship [22].

In our study, females had lighter valves than males when AVW was expressed in absolute values or adjusted for BSA, but not when was indexed for the aortic annulus cross-sectional area. This finding is in contrast with other reports $[13,18,23]$ but agrees with a recent study which determined that AVA/aortic annulus area (i.e., aortic valve calcium density) was similar in both genders; importantly, when compared with males, females had greater amounts of valvular fibrosis for the same hemodynamic stenosis severity [9].

The prevalence of BAV in this study is similar to that reported by Mohler et al. [18] (i.e. 11\%) using electron beam-computerized tomography (EBCT) for the determination of AVC load, but it is lower than in other studies where pathoanatomic diagnosis was used and a prevalence of $26-48 \%$ was reported $[16,17,21,24]$. In agreement with prior studies, we found that the bicuspid valves are heavier than the tricuspid valves [21]. This universally reported finding can be explained by the abnormal mechanical and shear forces exercised on the BAV, which are associated with increased oxidative stress and chronic inflammation that lead to valvular endothelial disruption and, ultimately, excess calcium deposition [25]. In addition, the BAV have larger cusps than the tricuspid ones, which may lead to different patterns of calcification, taking the form of macroaggregates, in contrast with the 
microaggregates in the tricuspid valves, where, compared with the BAV [16], a closer relationship between AVW with transvalvular gradients can be found.

It has been reported that the excised valve can be used as a surrogate for valve calcification [15], closely reflecting AS severity; a good correlation between AVW with the transvalvular peak velocity and gradients has been found $[14,16,22,26,27]$. In contrast, the correlation between AVW and AVA is less clear. With the exception of 1 study which used EBCT [12], the majority of investigators have reported no correlation/a weak correlation between AVC and AVA regardless of the method used for the determination of the AVC load (i.e., MDCT or pathology) $[14,16,26]$ or the AVA calculation (i.e., noninvasive or invasive). The determination of AVA is affected by inherent limitations of the continuity equation (i.e., the assumption of the LVOT circularity), accuracy of the Doppler recordings, variations in the stroke volume and vascular load (i.e., elevated systolic blood pressure), and inherent drawbacks of the Gorlin equation [28].

The analysis of the 4 hemodynamic groups of AS in the restricted cohort showed that patients with "classic" AS (i.e., NF/HG) had the heaviest valves. Compared with this group and regardless of flow, patients with low gradients had the lightest valves, in agreement with a number of reports indicating that severe AS with NF/LG and moderate AS are very similar [29], and possibly due to the strong correlation between the baseline gradients with AVC burden [27]. Among the 7 echocardiographic parameters analyzed, DOI was found to have the strongest correlation with AVW, possibly because it eliminated the need to measure LVOT diameter which has known drawbacks. In both low-gradient groups, very few or no correlations were found with AVW or adjusted AVW, especially in the LF/LG group (i.e., "paradoxical" low flow-low gradient AS) which reiterates the value of AVCload quantification in such patients [22].

The magnitude of AVC load has been evaluated in relation to outcome measures in several studies where AVR was taken as an outcome variable, and where the association of AVW and all-cause death was examined in only a limited number of patients [30, 31]. In these studies, a positive correlation was found between AVC load and mortality, possibly explained by left ventricular geometrical, morphologic, myocardial perfusion, and electrical abnormalities, in addition to altered vascular load and advanced patients' age (associated with severe atherosclerosis and multiple comorbidities). In this study, when AVW was analyzed as a categorical variable, no significant association with death was found in either gender, consis-

AVW and Indices of AS tent with the report by Roberts et al. [32] where an AVW of $4 \mathrm{~g}$ was chosen as the threshold between lighter and heavier valves. In contrast, in our study, when AVW was treated as a continuous variable, an inverse association with mortality was found in females only. We tried to understand this paradox by separately analyzing the clinical, hemodynamic, and echocardiographic data of both genders. Although older than males, females had a better medical history, LV function, and steady vascular load profile. In the entire cohort, a higher number of females than males were diagnosed with severe AS. Although we could not reliably assess patients' symptoms, considering that in females the valvular fibrotic changes might be the predominant mechanism of aortic valve orifice narrowing [9], it is possible that some females may have developed calcific valves. This led to the higher degree of AS severity, more symptoms, and earlier referral to AVR, ultimately increasing their survival. It has been shown that for a similar AVC load, women have a higher hemodynamic severity of AS than men [26]. Due to the curvilinear relationship between AVA and AVC load, a small AVC accumulation on a less calcified valve results in a large decrease in AVA [12]. Finally, we conducted a sensitivity test using the E-value. The E-value [33] is defined as the minimum strength of association on the risk ratio scale (i.e., the risk in exposed vs. unexposed), between an unmeasured confounder and treatment/outcome, to fully explain away a specific treatment/outcome association. This quantifies how susceptible the paradoxical inverse association of AVW with all-cause mortality in females could be, i.e., due to bias resulting from unmeasured confounding or measurement error. We found that the aforementioned HR of 0.668 (CI 0.471-0.946) has an E-value of 1.97 for the HR estimate and 1.24 for the CI. The minimum strength of unmeasured confounders on the HR scale would need to be 1.24 to alter the CI to null (to include 1 , making $p>0.05$ ), or 1.97 to make the HR itself 1.00. This is a rather small threshold to meet, suggesting that our paradoxical result was highly sensitive to small, unaccounted-for biases in this analysis.

\section{Strengths and Limitations}

To our knowledge, this series is the largest in which AVW measured after AVR was analyzed in relation to most of the echocardiographic indices of AS and all-cause mortality. Moreover, we separately analyzed each of the hemodynamic groups and showed, for the first time, a lack of correlation between AVW and echocardiographic indices in the low-gradient groups. The type of calcification (i.e., micro- vs. macroaggregates) and the amount of 
valvular fibrosis were not analyzed. To increase accuracy, we chose a restricted cohort with isolated severe AS and preserved LVEF; this precluded the correlation analyses in lower severities of AS. In addition, as in many retrospective studies, patients' symptoms could not be accurately ascertained. None of the patients had MDCT data for AVC quantification. Nevertheless, an excellent correlation between AVW and AVC by MDCT has been reported [15], and a regression equation translating AVC score by MDCT into AVW proposed (i.e., AVW = AVC score/1.7) [12]. Additionally, as frequently observed in tertiary referral centers, many patients were directly referred to surgery without a preoperative TTE being performed in our lab. In these patients, we used the TEE data recorded at the time of intraoperative study, which may have slightly underestimated the true magnitude of the transvalvular velocities/gradients. However, great care was taken when sampling the LVOT and recording the highest transvalvular velocities from the deep transgastric view, together with meticulous recording of the hemodynamic data.

In conclusion, we found a modest correlation between AVW and the echocardiographic indices of AS. This reflects the complex pathophysiology of this condition and the possible pitfalls of methods currently used for the evaluation of AS severity. The dimensionless index came out as the strongest correlate with the AVW, showing its prominence among the other hemodynamic echocardiographic indices employed in the clinical work. Regardless of flow magnitude, AVW was not associated with the echocardiographic variables in the low-gradient groups, thereby suggesting the diagnostic challenges presented by such patients and the need for multiparametric and, eventually, a multimodality approach in such cases. When analyzed as a categorical variable, in both genders, AVW had a neutral effect on survival. Future studies may validate our observations and contribute to the expansion of the current understanding of AS and its evaluation.

\section{Statement of Ethics}

The study was approved by the St. Francis Hospital Institutional Review Board.

\section{Disclosure Statement}

The authors have no conflicts of interest to declare.

\section{Funding Source}

This study was funded by the St. Francis Hospital Foundation.

\section{Author Contributions}

Jonathan Weber: data analysis/interpretation and drafting the article. Simcha Pollack: statistics. Florentina Petillo: data collection (echocardiography). Ana Anagnostopoulos: data collection (pathology). J. Jane Cao: critical revision of the article. Eddy Barasch: concept/design and approval of the article.

\section{References}

1 Thanassoulis G, Campbell CY, Owens DS, Smith JG, Smith AV, Peloso GM, et al.; CHARGE Extracoronary Calcium Working Group. Genetic associations with valvular calcification and aortic stenosis. N Engl J Med. 2013 Feb;368(6):503-12.

2 Probst V, Le Scouarnec S, Legendre A, Jousseaume V, Jaafar P, Nguyen JM, et al. Familial aggregation of calcific aortic valve stenosis in the western part of France. Circulation. 2006 Feb;113(6):856-60.

3 Le Gal G, Bertault V, Bezon E, Cornily JC, Barra JA, Blanc JJ. Heterogeneous geographic distribution of patients with aortic valve stenosis: arguments for new aetiological hypothesis. Heart. 2005 Feb;91(2):247-9.

4 Radhakrishna U, Albayrak S, Alpay-Savasan Z, Zeb A, Turkoglu O, Sobolewski P, et al. Genome-Wide DNA Methylation Analysis and Epigenetic Variations Associated with Congenital Aortic Valve Stenosis (AVS). PLoS One. 2016 May;11(5):e0154010.
5 Otto CM, Kuusisto J, Reichenbach DD, Gown $\mathrm{AM}, \mathrm{O}$ 'Brien KD. Characterization of the early lesion of 'degenerative' valvular aortic stenosis. Histological and immunohistochemical studies. Circulation. 1994 Aug;90(2):84453.

6 Stefenelli T, Mayr H, Bergler-Klein J, Globits S, Woloszczuk W, Niederle B. Primary hyperparathyroidism: incidence of cardiac abnormalities and partial reversibility after successful parathyroidectomy. Am J Med. 1993 Aug; 95(2): 197-202.

7 Ortlepp JR, Hoffmann R, Ohme F, Lauscher J, Bleckmann F, Hanrath P. The vitamin D receptor genotype predisposes to the development of calcific aortic valve stenosis. Heart. 2001 Jun;85(6):635-8.

8 Dweck MR, Boon NA, Newby DE. Calcific aortic stenosis: a disease of the valve and the myocardium. J Am Coll Cardiol. 2012 Nov; 60(19):1854-63.
9 Simard L, Côté N, Dagenais F, Mathieu P, Couture C, Trahan S, et al. Sex-Related Discordance Between Aortic Valve Calcification and Hemodynamic Severity of Aortic Stenosis: Is Valvular Fibrosis the Explanation? Circ Res. 2017 Feb;120(4):681-91.

10 Liu AC, Joag VR, Gotlieb AI. The emerging role of valve interstitial cell phenotypes in regulating heart valve pathobiology. Am J Pathol. 2007 Nov; 171(5):1407-18.

11 Kaden JJ, Freyer S, Weisser G, Willingstorfer W, Bilbal A, Pfleger S, et al. Correlation of degree of aortic valve stenosis by Doppler echocardiogram to quantity of calcium in the valve by electron beam tomography. Am J Cardiol. 2002 Sep;90(5):554-7.

12 Messika-Zeitoun D, Aubry MC, Detaint D, Bielak LF, Peyser PA, Sheedy PF, et al. Evaluation and clinical implications of aortic valve calcification measured by electron-beam computed tomography. Circulation. 2004 Jul; 110(3):356-62. 
13 Cueff C, Serfaty JM, Cimadevilla C, Laissy JP, Himbert D, Tubach F, et al. Measurement of aortic valve calcification using multislice computed tomography: correlation with haemodynamic severity of aortic stenosis and clinical implication for patients with low ejection fraction. Heart. 2011 May;97(9):721-6.

14 Roberts WC, Ko JM. Relation of weights of operatively excised stenotic aortic valves to preoperative transvalvular peak systolic pressure gradients and to calculated aortic valve areas. J Am Coll Cardiol. 2004 Nov;44(9): 1847-55.

15 Thaden JJ, Nkomo VT, Suri RM, Maleszewski JJ, Soderberg DJ, Clavel MA, et al. Sex-related differences in calcific aortic stenosis: correlating clinical and echocardiographic characteristics and computed tomography aortic valve calcium score to excised aortic valve weight. Eur Heart J. 2016;37:693-9.

16 Razzolini R, Longhi S, Tarantini G, Rizzo S, Napodano M, Abate E, et al. Relation of aortic valve weight to severity of aortic stenosis. Am J Cardiol. 2011 Mar;107(5):741-6.

17 Roberts WC, Ko JM, Hamilton C. Comparison of valve structure, valve weight, and severity of the valve obstruction in 1849 patients having isolated aortic valve replacement for aortic valve stenosis (with or without associated aortic regurgitation) studied at 3 different medical centers in 2 different time periods. Circulation. 2005 Dec;112(25):3919-29.

18 Mohler ER 3rd, Medenilla E, Wang H, Scott C. Aortic valve calcium content does not predict aortic valve area. J Heart Valve Dis. 2006 May;15(3):322-8.
19 Baumgartner H, Hung J, Bermejo J, Chambers JB, Edvardsen T, Goldstein S, et al. Recommendations on the Echocardiographic Assessment of Aortic Valve Stenosis: A Focused Update from the European Association of Cardiovascular Imaging and the American Society of Echocardiography. J Am Soc Echocardiogr. 2017 Apr;30(4):372-92.

20 VanderWeele TJ. Principles of confounder selection. Eur J Epidemiol. 2019 Mar;34(3): 211-9.

21 Roberts WC, Ko JM. Weights of operatively excised stenotic unicuspid, bicuspid, and tricuspid aortic valves and their relation to age, sex, body mass index, and presence or absence of concomitant coronary artery bypass grafting. Am J Cardiol. 2003 Nov;92(9):1057-65.

22 Clavel MA, Côté N, Mathieu P, Dumesnil JG, Audet A, Pépin A, et al. Paradoxical low-flow, low-gradient aortic stenosis despite preserved left ventricular ejection fraction: new insights from weights of operatively excised aortic valves. Eur Heart J. 2014 Oct;35(38):2655-62.

23 Thomassen HK, Cioffi G, Gerdts E, Einarsen E, Midtbø HB, Mancusi C, et al. Echocardiographic aortic valve calcification and outcomes in women and men with aortic stenosis. Heart. 2017 Oct;103(20):1619-24.

24 Sievers HH, Schmidtke C. A classification system for the bicuspid aortic valve from 304 surgical specimens. J Thorac Cardiovasc Surg. 2007 May;133(5):1226-33.

25 Manno G, Bentivegna R, Morreale P, Nobile D, Santangelo A, Novo S, et al. Chronic inflammation: a key role in degeneration of bicuspid aortic valve. J Mol Cell Cardiol. 2019 May;130:59-64.

26 Aggarwal SR, Clavel MA, Messika-Zeitoun D, Cueff C, Malouf J, Araoz PA, et al. Sex differences in aortic valve calcification measured by multidetector computed tomography in aortic stenosis. Circ Cardiovasc Imaging. 2013 Jan;6(1):40-7.
27 Stähli BE, Nguyen-Kim TD, Gebhard C, Frauenfelder T, Tanner FC, Nietlispach F, et al. Calcification Characteristics of LowFlow Low-Gradient Severe Aortic Stenosis in Patients Undergoing Transcatheter Aortic Valve Replacement. Cardiol Res Pract. 2015; 2015:802840.

28 Cannon SR, Richards KL, Crawford M. Hydraulic estimation of stenotic orifice area: a correction of the Gorlin formula. Circulation. 1985 Jun;71(6):1170-8.

29 Tribouilloy C, Rusinaru D, Maréchaux S, Castel AL, Debry N, Maizel J, et al. Low-gradient, low-flow severe aortic stenosis with preserved left ventricular ejection fraction: characteristics, outcome, and implications for surgery. J Am Coll Cardiol. 2015 Jan;65(1):55-66.

30 Rosenhek R, Binder T, Porenta G, Lang I, Christ G, Schemper M, et al. Predictors of outcome in severe, asymptomatic aortic stenosis. N Engl J Med. 2000 Aug;343(9):611-7.

31 Clavel MA, Pibarot P, Messika-Zeitoun D, Capoulade R, Malouf J, Aggarval S, et al. Impact of aortic valve calcification, as measured by MDCT, on survival in patients with aortic stenosis: results of an international registry study. J Am Coll Cardiol. 2014 Sep;64(12): 1202-13.

32 Roberts WC, Ko JM, Filardo G. Comparison of heavier versus lighter operatively excised stenotic aortic valves in adults with aortic stenosis and implications for percutaneous aortic valve implantation without replacement. Am J Cardiol. 2009 Aug;104(3):393-405.

33 VanderWeele TJ, Ding P. Sensitivity analysis in observational research: introducing the EValue. Ann Intern Med. 2017 Aug;167(4): 268-74. 